\title{
Linking chemical reactivity, magic numbers, and local electronic properties of clusters
}

\section{Citation}

Alfonso, Dominic R., Shi-Yu Wu, Chakram S. Jayanthi, and Efthimios Kaxiras. 1999. "Linking Chemical Reactivity, Magic Numbers, and Local Electronic Properties of Clusters." Physical Review B 59 (11): 7745-50. https://doi.org/10.1103/physrevb.59.7745.

\section{Permanent link}

http://nrs.harvard.edu/urn-3:HUL.InstRepos:41384077

\section{Terms of Use}

This article was downloaded from Harvard University's DASH repository, and is made available under the terms and conditions applicable to Other Posted Material, as set forth at http:// nrs.harvard.edu/urn-3:HUL.InstRepos:dash.current.terms-of-use\#LAA

\section{Share Your Story}

The Harvard community has made this article openly available.

Please share how this access benefits you. Submit a story.

Accessibility 


\title{
Linking chemical reactivity, magic numbers, and local electronic properties of clusters
}

\author{
Dominic R. Alfonso \\ Department of Physics, University of Louisville, Louisville, Kentucky 40292 \\ and Condensed Matter Section, International Centre for Theoretical Physics, Trieste I-34100, Italy \\ Shi-Yu Wu and Chakram S. Jayanthi \\ Department of Physics, University of Louisville, Louisville, Kentucky 40292 \\ Efthimios Kaxiras \\ Physics Department, Harvard University, Cambridge, Massachusetts 02138
}

(Received 24 August 1998)

\begin{abstract}
The interplay of local energetics, local electron occupancies, and local density of states is the key to the understanding of chemical reactivity. We define local measures, within a nonorthogonal tight-binding scheme, which clearly and unambiguously determine these local properties for an aggregate of atoms, such as a solid or a cluster. Using these measures, we identify the electronic level mechanisms responsible for the chemical reactivity of clusters of different sizes. A clear and concise picture of why $\mathrm{Si}_{33}$ is chemically inert while $\mathrm{Si}_{49 \mathrm{~A}}$ is reactive emerges from this analysis. A scheme for quantifying the dangling bonds is also presented in this work. [S0163-1829(99)01511-8]
\end{abstract}

\section{INTRODUCTION}

For systems with reduced or no symmetry, the interplay among various features associated with the local environments is the key in determining the global properties of a system. In situations where there is a redistribution of valence electrons and/or charge transfer, analysis of the local bonding and rebonding involved in the formation of defect configurations such as clusters, surfaces, and interfaces can provide invaluable information on the local electronic structure and the energetics. Such information is critical for the understanding of system properties at the local microscopic level.

In this paper, we use a local approach to investigate the existence of "magic numbers" in the chemical reactivity of Si clusters of intermediate sizes. ${ }^{1}$ The approach defines local measures for the electronic structure in the framework of a nonorthogonal tight-binding Hamiltonian (NOTB). Local measures used in the analysis include local orbital energy, bond energy, bond charge, orbital charge, and local density of states (LDOS). Since the NOTB Hamiltonian is related to linear combination of atomic orbitals (LCAO)-based methods, the formalism of local analysis used in this paper becomes identical to Mulliken's population analysis technique - a method well known in the quantum-chemistry community. $^{2}$ The validity of the local analysis technique depends crucially on the nature of the basis functions used to expand the wave function. In the NOTB approach, although the basis functions are not explicitly stated, they are assumed to be localized. This makes the NOTB approach reliable to extract local information at the microscopic level.

Our study of clusters in the intermediate range is based on structures derived from models constructed using the surface reconstruction induced geometries (SRIG) proposed by Kaxiras. $^{3,4}$ Of the clusters of different sizes that are compatible with the SRIG requirements and that are relatively stable (see Sec. III), only clusters with "magic" sizes 33, 39, and 45 would be expected to be chemically inert, while the rest (clusters with sizes 49, 57, and 61) would have much higher (by several orders of magnitude) reactivity, in agreement with experimental measurements for $\mathrm{Si}$ clusters in this range. ${ }^{1,5-8}$ This expectation is based on rather simple arguments related to the highest occupied molecular orbitallowest unoccupied molecular orbital (HOMO-LUMO) gap of these models. ${ }^{4}$ However, in order to establish this rigorously, a much more detailed analysis of the chemical reactivity properties of the models is required. Therefore, in this paper a detailed local analysis of the electronic structure based on the NOTB Hamiltonian has been carried out.

\section{METHODOLOGY}

For a semiempirical nonorthogonal tight-binding (NOTB) scheme, the Hamiltonian matrix elements $H_{i \alpha, j \beta}$ and the overlap matrix elements $S_{i \alpha, j \beta}$ are defined in terms of some finite set of local-basis functions $\left\{\varphi_{i \alpha}(\mathbf{r})\right\}$, which, as mentioned above, are not explicitly specified. Here $(i, \alpha)$ denotes the $\alpha$ orbital at site $i$. These matrix elements are in fact parametrized functions of $\mathbf{R}_{i j}=\mathbf{R}_{i}-\mathbf{R}_{j}$, where $\mathbf{R}_{i}$ is the position vector for site $i$. The parameters defining the matrix elements are usually constructed by fitting to an experiment and/or first-principles data base of properties of both bulk systems as well as clusters. In the tight-binding approach, the wave function $\Psi_{\lambda}$ of the system is expanded in terms of a finite set of local-basis function such that

$$
\Psi_{\lambda}=\sum_{i \alpha} C_{i \alpha, \lambda} \varphi_{i \alpha}
$$

When Eq. (1) is substituted into the Schrödinger equation of the system, the column vector of the coefficients of expansion $\mathbf{C}_{\lambda}$ will satisfy the general eigenvalue equation $H \mathbf{C}_{\lambda}$ $=E_{\lambda} \mathbf{S C}_{\lambda}$, where $E_{\lambda}$ is the electronic eigenenergy of the sys- 
tem, $\mathbf{C}_{\lambda}$ takes the role of the wave function, and $\mathbf{C}_{\lambda}^{\dagger} \mathbf{S C}_{\lambda}$ $=1$. In this scheme, the Hamiltonian and overlap matrix elements are defined by

$$
H_{i \alpha, j \beta}\left(\mathbf{R}_{i j}\right)=\int \varphi_{i \alpha}^{\star}(\mathbf{r}) H \varphi_{j \beta}(\mathbf{r}) \mathbf{d} r
$$

and

$$
S_{i \alpha, j \beta}\left(\mathbf{R}_{i j}\right)=\int \varphi_{i \alpha}^{\star}(\mathbf{r}) \varphi_{j \beta}(\mathbf{r}) \mathbf{d} r .
$$

The electronic band energy of the system in the nonorthogonal basis set is then

$$
\begin{aligned}
E_{\text {band }} & =2 \sum_{\lambda}^{\mathrm{occ}} E_{\lambda}=2 \sum_{\lambda}^{\mathrm{occ}}\left\langle\Psi_{\lambda}|H| \Psi_{\lambda}\right\rangle \\
& =2 \sum_{\lambda}^{\mathrm{occ}} \sum_{i \alpha, j \beta} C_{i \alpha, \lambda} C_{j \beta, \lambda} H_{j \beta, i \alpha} .
\end{aligned}
$$

Similarly, the number of electrons in the system is given by

$$
N=2 \sum_{\lambda}^{\text {occ }}\left\langle\Psi_{\lambda} \mid \Psi_{\lambda}\right\rangle=2 \sum_{\lambda}^{\text {occ }} \sum_{i \alpha, j \beta} C_{i \alpha, \lambda} C_{j \beta, \lambda} S_{j \beta, i \alpha} .
$$

For the tight-binding Hamiltonians, the total energy of a system is given by $E_{\text {tot }}=E_{\text {band }}+E_{\text {rep }}$, where $E_{\text {rep }}$ is the sum of pairwise repulsive terms, which are also obtained by fitting. Equations (3) and (4) can be rewritten as $E_{\text {band }}=\Sigma_{i} E_{i}$, and $N=\Sigma_{i} N_{i}$, with the site energy $E_{i}$ and the local electron number $N_{i}$ given by

$$
\begin{gathered}
E_{i}=\sum_{\alpha} \gamma_{i \alpha, i \alpha} \epsilon_{i \alpha}+\sum_{(j \neq i) \alpha \beta} \gamma_{i \alpha, j \beta} H_{j \beta, i \alpha}, \\
N_{i}=\sum_{\alpha} \gamma_{i \alpha, i \alpha}+\sum_{(j \neq i) \alpha \beta} \gamma_{i \alpha, j \beta} S_{j \beta, i \alpha},
\end{gathered}
$$

where $\gamma_{i \alpha, j \beta}=2 \sum_{\lambda}^{\mathrm{occ}} C_{i \alpha, \lambda} C_{j \beta, \lambda}$. In deriving Eqs. (5) and (6), we have used $H_{i \alpha, i \beta}=\epsilon_{i \alpha} \delta_{\alpha, \beta}$ and $S_{i \alpha, i \beta}=\delta_{\alpha, \beta}$. Equation (5) permits the evaluation of the site energy in terms of the local promotional/demotional energy $\left(\gamma_{i \alpha, i \alpha} \epsilon_{i \alpha}\right)$ and the bonding energies $\left(\gamma_{i \alpha, j \beta} H_{j \beta, i \alpha}\right)$ between the atom at site $i$ and its neighbors, while Eq. (6) allows the determination of the number of electrons at a given site in terms of the number of electrons occupying local orbitals $\left(\gamma_{i \alpha, i \alpha}\right)$ and those shared by orbitals of neighboring sites $\left(\gamma_{i \alpha, j \beta} S_{j \beta, i \alpha}\right)$. The information obtained from the local analysis of the energetics and that from the local analysis of electron occupancy are complementary to each other. When combined, they give a transparent picture of the nature of local electronic structure and how it affects the energetics, and thus other relevant properties of the system under study.

Equations (1)-(6) provide the framework for the local analysis of the electronic structure that is common to all LCAO-based approaches as first demonstrated by Mulliken. ${ }^{2}$ We would, however, like to caution on the validity of assigning local physical quantities to different atomic sites based on Eqs. (5) and (6). If the basis functions $\varphi_{i \alpha}$ 's are very diffuse, there is a degree of arbitrariness in such assignments. This is because for diffuse basis functions, most of their weight is at distances away from the sites where they are centered. On the other hand, if $\varphi_{i \alpha}$ 's are localized orbitals such as in the case of NOTB approach where the orbitals are implicitly implied to be localized, it is clearly reasonable to assign local physical quantities to the sites where the orbitals are centered.

\section{LOCAL ANALYSIS OF CHEMICAL REACTIVITY OF SILICON CLUSTERS}

To address issues involved in the existence of magic numbers ${ }^{1}$ in the chemical reactivity of Si clusters of intermediate sizes, one has to determine first the stable configurations for the clusters in this range. The determination of the lowest energy structures of these clusters through full exploration of their configurational phase spaces via firstprinciples calculations is a difficult computational task. An alternate procedure to study these systems has been introduced by Kaxiras. ${ }^{3,4}$ The procedure is based on the fact that for $\mathrm{Si}$ clusters of sizes ranging from a few tens to a few hundreds of atoms, the cluster geometries are expected to be dominated to a large extent by their surface features. Using the requirement that all interior cluster atoms are bulklike, and all exterior atoms take on features associated with the most stable reconstructions of $\mathrm{Si}$, it was found that only a few cluster sizes $(33,39 A, 39 B, 45 A, 45 B, 49 A, 49 B, 57$, $61 A$, and $61 B$ ) are compatible with these criteria. ${ }^{4}$ The models obtained in this way are referred as surface reconstruction induced geometries (SRIG). These models have been shown to be successful in understanding mobility measurements., ${ }^{3,4}$ Labels $A$ and $B$ used above refer to two different structures with the same cluster size which satisfy the SRIG requirements. The bonds associated with exterior atoms in SRIG models are generally under stress with the typical angles between these bonds considerably distorted relative to the tetrahedral angle. In Ref. 4, the optimized structures of SRIG models were determined by minimizing the magnitude of the Helmann-Feynmann forces under the constraint that the clusters have overall tetrahedral symmetry. It was, however, recognized that stable equilibrium structures with characteristics similar to SRIG models but lower symmetry may exist once the constraint is relaxed. We based our search for the stable structures of Si clusters of intermediate size on SRIG models. We utilized the NOTB Hamiltonian for Si constructed by Menon and Subbaswamy ${ }^{9}$ because of its remarkable success in predicting structural properties of small clusters. In our study, we first verify the reliability of the Hamiltonian by using it to obtain the optimized SRIG structures. The results are in total agreement with those obtained by the densityfunctional theory (DFT)/local-density approximation (LDA) calculations of Ref. 4. To obtain the equilibrium geometries, we start from the DFT/LDA-optimized configurations and allow the atoms to relax without the constraint of tetrahedral symmetry using the dynamical quenching technique ${ }^{10}$ until the forces on all atoms converged to $\sim 0.01 \mathrm{eV} / \mathrm{A}$. The resulting equilibrium structures possess geometrical characteristics very similar to the corresponding SRIG models. They also have binding energies ( $\sim 80 \%$ of the bulk value) and cross sections similar to the corresponding results of the SRIG models obtained by the DFT/LDA scheme. ${ }^{4}$ The trend in the relative stabilities is likewise generally well repro- 


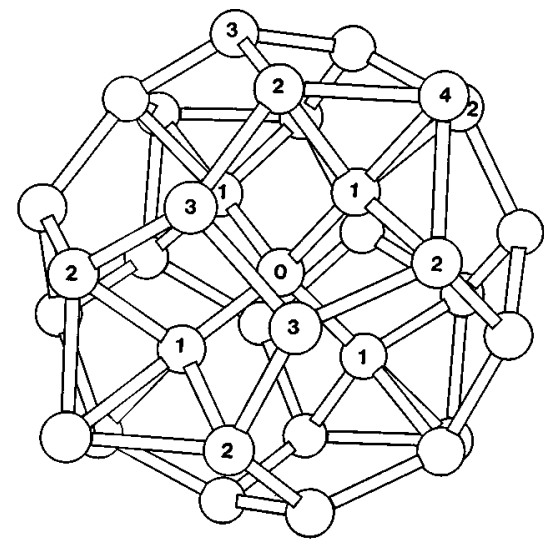

(a)

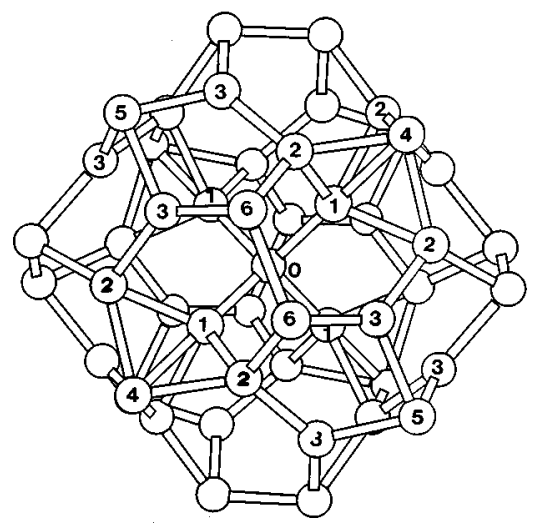

(b)

FIG. 1. Stable structures of (a) $\mathrm{Si}_{33}$ and (b) $\mathrm{Si}_{49 A}$, respectively.

duced with the exception of cluster $61 A \cdot{ }^{4}$ In the case of $61 A$, the relaxation without the constraint of overall tetrahedral symmetry has led to a more compact structure that has the lowest energy in the sequence of structures based on SRIG models.

To understand the difference in the chemical reactivities of these clusters, in particular, why the $\mathrm{Si}_{33}$ cluster is chemi- cally inert and $\mathrm{Si}_{49 \mathrm{~A}}$ is reactive, we have performed the local analysis of the structural, energetic and electronic properties of these two SRIG-based clusters. The key ingredients for the local analysis are $n_{i \alpha}=\gamma_{i \alpha, i \alpha}$ (the electron occupancy at $i \alpha), N_{\text {bond }}^{(i j)}=\Sigma_{\alpha \beta}\left\{\gamma_{i \alpha, j \beta} S_{j \beta, i \alpha}+\gamma_{j \beta, i \alpha} S_{i \alpha, j \beta}\right\} \quad$ [electrons in the ( $i$-j) bond region], $N_{i}=\Sigma_{\alpha} n_{i \alpha}+\Sigma_{j \neq i} \frac{1}{2} N_{\text {bond }}^{(i j)}$ (electrons at site $i)$, and $E_{\text {bond }}=\Sigma_{\alpha \beta}\left\{\gamma_{i \alpha, j \beta} H_{j \beta, i \alpha}+\gamma_{j \beta, i \alpha} H_{i \alpha, j \beta}\right\}$. The bond energy $\left(E_{\text {bond }}\right)$ can be further broken into four contributions arising from $V_{s s \sigma}, V_{s p \sigma}, V_{p p \sigma}$, and $V_{p p \pi}$ interactions to shed light on the nature of the bonding. ${ }^{11}$ Results of our local analysis of electronic properties for clusters $\mathrm{Si}_{33}$ [Fig. 1(a)] and $\mathrm{Si}_{49 \mathrm{~A}}$ [Fig. 1(b)] are given in Tables I and II. The equilibrium structures for these clusters are obtained by the molecular dynamics-based "simulated-annealing" procedure discussed above. From Fig. 1 it can be seen that the equilibrium structures for $\mathrm{Si}_{33}$ and $\mathrm{Si}_{49 A}$ are only slightly distorted from their corresponding optimized SRIG models. There are five types of equivalent atoms in $\mathrm{Si}_{33}$ (labeled 0 and 1 for interior atoms and 2-4 for exterior atoms) and seven types in $\mathrm{Si}_{49 A}$ (2-6 for exterior atoms). The five interior atoms $(0,1)$ in both structures are coordinated in a tetrahedral fashion forming bonds (0-1) that are predominantly $\sigma$ in character. These bonds are highly directional (typical of covalent bonds) as evidenced by the dominance of $\operatorname{sp} \sigma$ and pp $\sigma$ over ss $\sigma$ contribution to the bonding energy (see Table I). The bonds associated with exterior atoms are also directional and mainly $\sigma$ in nature. Notice also that, for most cases, the ss $\sigma$ contribution has antibonding character. In Table I, we have also given the results for $N_{\text {bond }}, E_{\text {bond }}$, and $r$, the bond length for various pairs of atoms. One can see a strong correlation between $N_{\text {bond }}$ and $E_{\text {bond }}$, i.e., the stronger the bond energy, the higher the charge accumulation in the bond region. We propose that the criterion for the presence of a bond can be determined according to $N_{\text {bond }} \geqslant 0.04$ ( $1 \%$ of valence electrons). Compared to the distance between atoms, this is a more reliable quantity in determining the pres-

TABLE I. A comparison of $s s \sigma, s p \sigma, p p \sigma$, and $p p \pi$ contributions to the bond energy ( $\left.E_{\text {bond }}\right)$, number of electrons in the bond region, and bondlengths $(r)$ for the nonequivalent bonds of $\mathrm{Si}_{33}, \mathrm{Si}_{49 A}$, and bulk diamond. Results obtained with the inclusion of electron correlations are shown in parentheses. The exterior atoms of $\mathrm{Si}_{33}$ are labeled 2 to 4 , while those of $\mathrm{Si}_{49 \mathrm{~A}}$ are labeled 2 to 6.

\begin{tabular}{lcccccccc}
\hline \hline \multirow{2}{*}{} & Bond & $E_{\text {bond }}^{s s \sigma}(\mathrm{eV})$ & $E_{\text {bond }}^{s p \sigma}(\mathrm{eV})$ & $E_{\text {bond }}^{p p \sigma}(\mathrm{eV})$ & $E_{\text {bond }}^{p p \pi}(\mathrm{eV})$ & $E_{\text {bond }}(\mathrm{eV})$ & $N_{\text {bond }}$ & $r(\AA)$ \\
\hline $\mathrm{Si}_{33}$ & $(0-1)$ & $-0.92(-0.80)$ & $-4.61(-4.52)$ & $-3.75(-3.83)$ & $-0.54(-0.56)$ & $-9.82(-9.71)$ & $0.54(0.53)$ & $2.24(2.24)$ \\
& $(1-2)$ & $0.10(0.14)$ & $-2.53(-2.54)$ & $-4.38(-4.41)$ & $-0.36(-0.36)$ & $-7.16(-7.17)$ & $0.42(0.42)$ & $2.32(2.32)$ \\
& $(1-4)$ & $0.53(0.53)$ & $-1.00(-0.96)$ & $-2.36(-2.34)$ & $-0.24(-0.22)$ & $-3.07(-2.98)$ & $0.19(0.18)$ & $2.44(2.44)$ \\
& $(2-3)$ & $-0.02(-0.04)$ & $-3.37(-3.40)$ & $-4.01(-4.00)$ & $-0.80(-0.79)$ & $-8.21(-8.23)$ & $0.45(0.45)$ & $2.40(2.40)$ \\
& $(2-4)$ & $0.16(0.14)$ & $-2.33(-2.34)$ & $-3.39(-3.38)$ & $-0.40(-0.39)$ & $-5.96(-5.96)$ & $0.32(0.32)$ & $2.49(2.50)$ \\
& $(3-3)$ & $0.08(0.08)$ & $-3.27(-3.63)$ & $-4.36(-4.36)$ & $-0.90(-0.89)$ & $-8.81(-8.81)$ & $0.49(0.49)$ & $2.37(2.37)$ \\
$\mathrm{Si}_{49 A}$ & $(0-1)$ & $-0.99(-0.89)$ & $-4.28(-4.13)$ & $-3.18(-3.29)$ & $-0.54(-0.63)$ & $-8.99(-8.94)$ & $0.46(0.46)$ & $2.32(2.34)$ \\
& $(1-2)$ & $-0.32(-0.30)$ & $-3.01(-3.02)$ & $-4.33(-4.32)$ & $-0.48(-0.67)$ & $-8.14(-8.09)$ & $0.45(0.45)$ & $2.36(2.36)$ \\
& $(1-4)$ & $0.61(0.57)$ & $-0.10(-0.08)$ & $-1.96(-1.77)$ & $0.19(0.19)$ & $-1.26(-1.09)$ & $0.09(0.08)$ & $2.60(2.63)$ \\
& $(2-3)$ & $-0.49(-0.40)$ & $-4.00(-3.87)$ & $-4.15(-4.21)$ & $-0.73(-0.75)$ & $-9.37(-9.23)$ & $0.51(0.50)$ & $2.34(2.35)$ \\
& $(2-4)$ & $-0.20(-0.14)$ & $-3.19(-3.06)$ & $-3.59(-3.69)$ & $-0.60(-0.52)$ & $-7.58(-7.40)$ & $0.41(0.40)$ & $2.39(2.40)$ \\
& $(2-6)$ & $-0.12(-0.13)$ & $-3.47(-3.54)$ & $-4.42(-4.30)$ & $-0.54(-0.58)$ & $-8.55(-8.55)$ & $0.47(0.47)$ & $2.37(2.37)$ \\
& $(3-5)$ & $-0.15(-0.14)$ & $-3.36(-3.32)$ & $-4.96(-4.92)$ & $-0.82(-0.83)$ & $-9.29(-9.21)$ & $0.53(0.53)$ & $2.34(2.34)$ \\
& $(3-6)$ & $-0.04(-0.03)$ & $-3.47(-3.41)$ & $-4.70(-4.68)$ & $-1.05(-1.15)$ & $-9.26(-9.27)$ & $0.53(0.53)$ & $2.34(2.34)$ \\
& $(6-6)$ & $0.44(0.44)$ & $-3.22(-3.62)$ & $-4.89(-4.50)$ & $-0.29(-0.26)$ & $-7.96(-7.94)$ & $0.45(0.45)$ & $2.40(2.38)$ \\
diamond & & -0.62 & -3.94 & -4.64 & -0.23 & -9.48 & 0.51 \\
\hline \hline
\end{tabular}


TABLE II. Site index (i), coordination (c), on-site orbital occupancy $\left(n_{i \alpha}, \alpha=s, p_{x}, p_{y}\right.$, and $\left.p_{z}\right)$, total number of on-site electrons ( $\left.N_{i}\right)$, ratio of the number of electrons in the bond region $(i-j)$ to the number of electrons associated with the site $i$, and local orbital energy $\left(E_{\text {lorb }}\right)$ are given for the bulk diamond, and for exterior atoms of $\mathrm{Si}_{33}$ and $\mathrm{Si}_{49 A}$. Results obtained with the inclusion of electron correlations are shown in parentheses.

\begin{tabular}{|c|c|c|c|c|c|c|c|c|c|}
\hline & $\mathrm{i}$ & $\mathrm{c}$ & $n_{i s}$ & $n_{i p x}$ & $n_{i p y}$ & $n_{i p z}$ & $N_{i}$ & $\sum_{j \neq i} \frac{N_{\text {bond }}^{(i j)}}{2 N_{i}}$ & $E_{\text {lorb }}(\mathrm{eV})$ \\
\hline \multirow[t]{3}{*}{$\mathrm{Si}_{33}$} & 2 & 4 & $1.48(1.48)$ & $0.63(0.61)$ & $0.63(0.61)$ & $0.56(0.55)$ & $4.11(4.06)$ & $20 \%(20 \%)$ & $-31.89(-31.38)$ \\
\hline & 3 & 3 & $1.56(1.55)$ & $0.56(0.56)$ & $0.56(0.56)$ & $0.66(0.67)$ & $4.04(4.03)$ & $17 \%(17 \%)$ & $-32.66(-32.54)$ \\
\hline & 4 & 4 & $1.76(1.76)$ & $0.54(0.55)$ & $0.54(0.55)$ & $0.54(0.55)$ & $3.96(3.98)$ & $14 \%(14 \%)$ & $-34.48(-34.73)$ \\
\hline \multirow[t]{4}{*}{$\mathrm{Si}_{49 \mathrm{~A}}$} & 3 & 3 & $1.42(1.45)$ & $0.59(0.62)$ & $0.39(0.44)$ & $0.49(0.55)$ & $3.67(3.83)$ & $21 \%(20 \%)$ & $-28.73(-30.54)$ \\
\hline & 4 & 4 & $1.61(1.60)$ & $0.50(0.63)$ & $0.50(0.63)$ & $0.50(0.63)$ & $3.78(3.88)$ & $17 \%(17 \%)$ & $-31.70(-33.12)$ \\
\hline & 5 & 3 & $1.61(1.65)$ & $0.68(0.63)$ & $0.68(0.63)$ & $0.68(0.63)$ & $4.44(4.26)$ & $18 \%(19 \%)$ & $-35.16(-33.01)$ \\
\hline & 6 & 3 & $1.64(1.61)$ & $0.66(0.61)$ & $0.86(0.80)$ & $0.57(0.52)$ & $4.44(4.25)$ & $16 \%(17 \%)$ & $-35.79(-33.48)$ \\
\hline diamond & & 4 & 1.24 & 0.59 & 0.59 & 0.59 & 4.0 & $25 \%$ & -28.23 \\
\hline
\end{tabular}

ence of a bond as will be seen in the next paragraph.

The atoms (4) at the apexes in both structures resemble the adatoms in the $\mathrm{Si}(111)-7 \times 7$ reconstruction ${ }^{12}$ and each is surrounded by three surface atoms (2) and forms predominantly $\sigma$ bonds. These apex atoms are directly above the interior atoms (1). In $\mathrm{Si}_{33}, r_{41}$ is less than $r_{42}$ (in both the LDA-optimized SRIG structure and our equilibrated structure). Yet the bond energy for 4-1 is only half of that of 4-2. The underlying reason is due to the fact that $N_{\text {bond }}$ for $4-1$ is about half of that of 4-2. The surface atoms (3) in $\mathrm{Si}_{33}$ relax to form dimers reminiscent of those formed in the reconstructed $\mathrm{Si}(100)$ surface. ${ }^{13}$ These bonds and those resulting from the interaction of each "dimer" atom with two other surface atoms (2) have strong $\pi$ character as evidenced from the $p p \pi$ contribution. In $\mathrm{Si}_{49 A}$, chain structures 3-5-3-6 of exterior atoms with strong $\pi$ character are in evidence. The chain structures are similar to the $2 \times 1 \pi$-bonded chain on the $\mathrm{Si}(111)$ surface. ${ }^{14}$ These surfacelike features of the exterior atoms in the Si clusters are apparently responsible for the unusual stability of SRIG-based equilibrium structures. The stability of the clusters is governed by a delicate balance between maximizing bond overlaps of neighboring atoms and minimizing the energy lost due to the redistribution of valence electrons among the orbitals and the migration of electrons to the bonding region. In the stable clusters, there is in general charge transfer from the interior region to the exterior region, resulting in an increase in the local orbital energy, $E_{\text {lorb }}=\gamma_{i s, i s} \epsilon_{s}+\left(\gamma_{i p_{x}, i p_{x}}+\gamma_{i p_{y}, i p_{y}}+\gamma_{i p_{z}, i p_{z}}\right) \epsilon_{p}$, for atoms in the interior region. The more "positive" $E_{\text {lorb }}$ in the interior region, however, is compensated by the more "negative' $E_{\text {lorb }}$ in the exterior region.

To shed light on how the interplay among the local orbital electron occupancy, the bond charge, the bond energy, and the local orbital energy affects the stability and structural properties of a cluster, we compare these properties of the two clusters $\left(\mathrm{Si}_{33}\right.$ and $\left.\mathrm{Si}_{49 A}\right)$ with the corresponding properties of the bulk $\mathrm{Si}$ in its most stable diamond phase. From Table I, it can be seen that, with the exception of $0-1$ bond in $\mathrm{Si}_{33}$, the bond energy of all the other bonds in both structures is higher (more positive) than the bond energy of the tetrahedral bond of the diamond phase of bulk Si, indicating that the strength of these bonds is weaker than the optimal cova- lent bond of bulk Si. A closer examination of the nature of bonding in the clusters when compared with the bonding in the diamond phase reveals that the most dramatic change occurs in $s s \sigma$ and $p p \pi$ bonding characteristics. A pattern indicating a reduction of $s s \sigma$ characteristics and an enhancement of $p p \pi$ characteristics in all the bonds when the bonding environment is distorted from the optimal tetrahedral bonding configuration emerges. This effect is particularly pronounced for the exterior ("surface") atoms in the clusters as their bonding configuration is considerably distorted from the normal tetrahedral configuration. The enhancement of the p $\pi$ characteristics in the bonds associated with the exterior atoms suggests that the "exterior" electrons are less localized and take on the character reminiscent of the metallic effect of the electrons on the surface of bulk Si. The reduction of $s s \sigma$ characteristics in the bond is an indication of less participation of $s$ electrons in the bond region as evidenced by the less negative $s s \sigma$ bond energy and, in some cases, even positive $s s \sigma$ bond energy (antibonding character) in the bonds associated with exterior atoms. From Table II, it is seen that the electron on-site occupancy for the $s$ orbital of the exterior atoms is considerably greater than the corresponding case of the bulk $\mathrm{Si}$, indicating the redistribution of $s$ electron from the bonding region into the on-site $s$ orbital and thus causing the reduction of $s s \sigma$ character in the bond. This redistribution of electrons into the on-site $s$ orbital also enhances the $E_{\text {lorb }}$ (more negative) of the exterior atoms as can be seen from Table II. It is this enhancement of the $E_{\text {lorb }}$, which contributes to the stability of the exterior atoms even though the bonding strength between them and their neighbors might be weaker than the normal tetrahedral bonding configuration. Thus, the combined effect of the redistribution into the on-site $s$ orbital and the enhancement of the $p p \pi$ bonding characteristics is the main factor, which determines the stability and structural properties of the two cluster structures.

The notion of "dangling", bonds has been frequently invoked to explain the energetics of the formation of defect configurations or surface effects. An explicit quantitative means to characterize the dangling bond is difficult to determine, and is lacking in many theoretical approaches. Within the framework of our local analysis, such a measure can be 
conveniently set up so that it provides not only a quantitative means to characterize dangling bonds but also a way to describe various characteristics of these bonds. Using bulk $\mathrm{Si}$ in the diamond lattice as the reference, we have determined that of the four electrons per atom in bulk $\mathrm{Si}$, one electron is shared uniformly by the four equivalent bonds formed between a Si atom and its four nearest neighbors. This suggests that the saturation of the bonding corresponds to a ratio of $\sum_{j \neq i} \frac{1}{2} N_{\text {bond }}^{(i j)} / N_{i}=25 \%$. In Table II, this ratio for exterior atoms in both structures range from $14 \%$ to $21 \%$, indicating the existence of dangling bonds associated with the corresponding "sites." Furthermore, for exterior atoms with equivalent dominant bonds, its electron occupancies in $p_{x}$, $p_{y}$, and $p_{z}$ orbitals are the same while for those with nonequivalent bonds, the electron occupancies in $p_{x}, p_{y}$, and $p_{z}$ orbitals are nonuniform. Thus, in the former case, the possible saturation of the "dangling" bonds is directed along the (111) direction while in the latter case, the saturation of the dangling bond favors the direction defined by the nonspherical charge distribution, i.e., $\left(n_{p_{x}}, n_{p_{y}}, n_{p_{z}}\right)$. In this way, a quantitative characterization is provided which leads to a vivid pictorial representation of the "dangling" of the unsaturated bond.

The possible reactive sites on the "surface" of a cluster are those exterior atoms that have substantial deviation from the normal electron occupancy $\left(N_{i}=4\right)$. From Table II, it can be seen that $N_{i}$ associated with the exterior atoms in $\mathrm{Si}_{33}$ does not deviate very much from 4 . Hence, it is not surprising that $\mathrm{Si}_{33}$ is chemically inert. On the other hand, in the case of $\mathrm{Si}_{49 \mathrm{~A}}$, the exterior atoms have significant probability to initiate reaction on its surface since their electron concentrations are either considerably lower (atoms 3 and 4) or higher (atoms 5 and 6) than 4. To further identify the most active sites, we have calculated the local density of states (LDOS) as a function of the eigenenergy $E_{\lambda}$ for these atoms. The results are shown in Fig. 2. It can be seen that, although both atoms 5 and 6 have $N_{i}$ substantially greater than 4 , only atoms of type 6 have pronounced features at and below the Fermi energy. This is a reflection of the propensity of atoms of type 6 to donate electrons to form bonds with electropositive reactants as compared to atoms of type 5 . On the other hand, the "electron-poor" atoms of type 4 have more pronounced features above the Fermi level than atoms of type 3, indicating that they have a higher affinity to accept electrons from reactants.

The charge transfers at exterior atoms in $\mathrm{Si}_{49 \mathrm{~A}}$ are overestimated (Table II). These can be attributed to the fact that electron correlation has not been accounted for in the NOTB Hamiltonian. We performed self-consistent calculations by incorporating a Hubbardlike term in the on-site NOTB

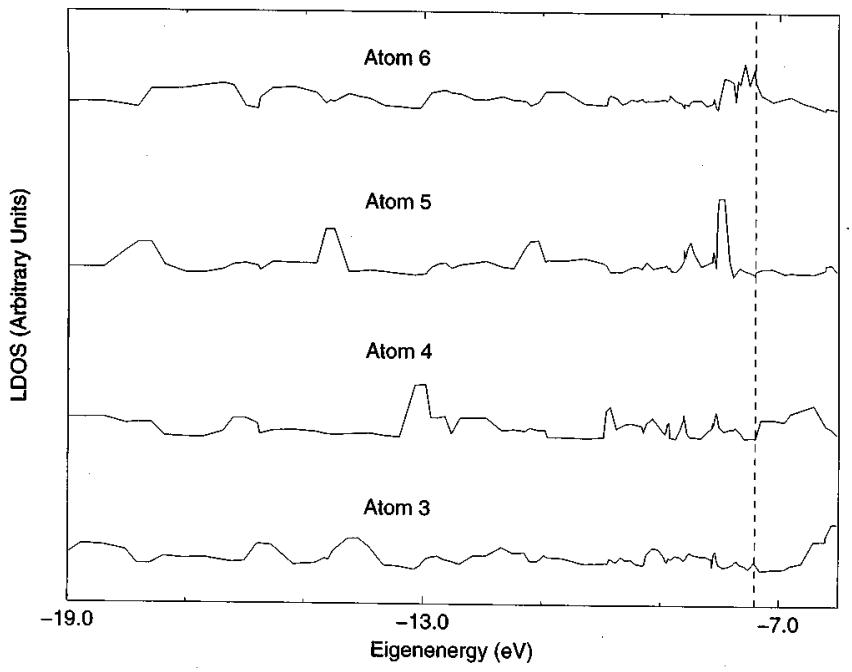

FIG. 2. Local density of states as a function of eigenenergy at the reactive sites $\left(3,4,5\right.$, and 6) of $\mathrm{Si}_{49 A}$. The Fermi level is indicated by the vertical dashed line.

Hamiltonian matrix elements. This term is given by $U\left(N_{i}\right.$ $-Z_{i}$ ), where $Z_{i}$ is the number of valence electrons of an isolated $\mathrm{Si}$ atom $\left(Z_{i}=4\right)$. The result yielded dramatic improvement of the charge transfer for the exterior atoms of $\mathrm{Si}_{49 A}$ as can be seen from Table II, where the results with the inclusion of Hubbard $U$ term are shown in parentheses. However, it did not alter the main conclusions of the present work. For instance, the structural properties, bonding nature, and chemical reactivities of the SRIG-based models do not deviate significantly from the case where no electron correlation effect is included.

\section{SUMMARY}

In summary, we have demonstrated how the interplay between the local electronic structure and energetics determines the chemical reactivity of Si clusters. More generally, we have shown the usefulness of the method of local analysis based on a NOTB Hamiltonian to study properties of complex systems with reduced symmetry.

\section{ACKNOWLEDGMENTS}

This work received support from the NSF through Grants No. EPS-9452895 and DMR-980274. One of us (C.S.J.) would like to acknowledge the support received from the Research Corporation under the Cottrell College Science award.
${ }^{1}$ See, for example, J. L. Elkind, J. M. Alford, F. D. Weiss, R. T. Laaksonen, and R. E. Smalley, J. Chem. Phys. 87, 2397 (1987).

${ }^{2}$ R. S. Mulliken, J. Chem. Phys. 23, 1833 (1955).

${ }^{3}$ E. Kaxiras, Chem. Phys. Lett. 163, 323 (1989); Phys. Rev. Lett. 64, 551 (1990)

${ }^{4}$ E. Kaxiras, Phys. Rev. B 56, 13455 (1997).
${ }^{5}$ U. Ray and M. F. Jarrold, J. Chem. Phys. 93, 5709 (1990); M. F. Jarrold, Y. Ijiri, and U. Ray, ibid. 94, 3607 (1991).

${ }^{6}$ M. F. Jarrold, J. E. Bower, and K. M. Creegan, J. Chem. Phys. 90, 3615 (1989); L. R. Anderson, S. Maruyama, and R. E. Smalley, Chem. Phys. Lett. 176, 348 (1991).

${ }^{7}$ M. F. Jarrold, U. Ray, and K. M. Creegan, J. Chem. Phys. 93, 224 
(1990); J. E. Bower and M. F. Jarrold, ibid. 97, 8312 (1992).

${ }^{8}$ S. Maruyama, L. R. Anderson, and R. E. Smalley, J. Chem. Phys. 93, 5349 (1990); J. M. Alford, R. T. Laaksonen, and R. E. Smalley, ibid. 94, 2618 (1991).

${ }^{9}$ M. Menon and K. R. Subbaswamy, Phys. Rev. B 50, 11577 (1994).

${ }^{10}$ M. P. Allen and D. J. Tildesley, Computer Simulation of Liquids (Oxford University Press, Oxford, 1987).
${ }^{11}$ A. T. Paxton and A. P. Sutton, J. Phys. C 20, L263 (1987).

${ }^{12}$ K. Takayanagi, Y. Tanishiro, M. Takahashi, and S. Takahashi, Surf. Sci. 164, 367 (1985).

${ }^{13}$ P. Krueger and J. Pollmann, Phys. Rev. Lett. 74, 1155 (1995).

${ }^{14}$ K. C. Pandey, Phys. Rev. Lett. 47, 223 (1981).

${ }^{15}$ J. C. Phillips, Phys. Rev. B 47, 14132 (1993); J. C. Grossman and L. Mitas, Phys. Rev. Lett. 74, 1323 (1995). 